\title{
Correlation between Overactive Bladder Syndrome and Severity of Coronary Artery Disease in Postmenopausal Women
}

\author{
Bahar Yuksel ${ }^{1}$, Faruk Özgör ${ }^{2}$, Murat Şahan², Semih Ozturk ${ }^{3}$, Mehmet Mustafa Can $^{3}$ and Omer Sarilar ${ }^{1}$ \\ ${ }^{1}$ Department of Obstetrics and Gynecology, Esenler Maternity and Children's Hospital, Istanbul, Turkey \\ ${ }^{2}$ Department of Urology, Haseki Teaching and Research Hospital, Istanbul, Turkey \\ ${ }^{3}$ Department of Cardiology Surgery, Haseki Teaching and Research Hospital, Istanbul, Turkey
}

\begin{abstract}
Objective: To analyse the correlation of overactive bladder (OAB) and severity of coronary artery disease (CAD) by using Gensini score in postmenopausal women.

Study Design: Observational correlation study design.

Place and Duration of Study: Haseki Training and Research Hospital, Istanbul, Turkey, from January 2016 to January 2018.

Methodology: Female patients in postmenopausal status, who had undergone coronary angiography due to CAD in tertiary academic care centre cardiology unit, were enrolled in the study. The Gensini score of each patient was calculated by two different cardiologists and the average score was taken. All participants filled the OAB-V8 questionnaire (Validated Turkish form). The participants were separated into two groups according to the severity OAB symptoms. Patients with OAB-V8 score $\geq 8$, were included into $\mathrm{OAB}$ Group and patients with OAB-V8 score <8, were enrolled into group 2 Non-OAB Group. Two groups were compared according to patients' demographic characteristics, Gensini scores, OAB-V8 scores, blood test outcomes, urinary system ultrasound, and uroflowmetry findings.

Results: One hundred and two patients with $O A B \geq 8$ score and 71 patients with $O A B<8$ score were compared. The median $B M I$ was $28.7(27.3-32.7) \mathrm{kg} / \mathrm{m}^{2}$ in OAB group and $27.5(27.0-31.1) \mathrm{kg} / \mathrm{m}^{2}$ in non-OAB group $(p=0.150)$. Additionally, OAB-V8 score and Gensini score were significantly higher in OAB group, compared to non-OAB group (25.0 (15.0-28.3) vs 4.0 (3.0-5.0), p<0.001 and 32.5 (27.8-42.7) vs. 10.0 (10.0-12.0), p<0.001, respectively). In blood chemistry, LDL level was the only marker found significantly different between groups and was significantly higher in patients with OAB (120.0 (90.0-148.5) mg/dl vs 97.0 (70.0-125.0) mg/dl, $p<0.001)$. Multivariate regression analysis revealed that Gensini score $\geq 20$ was an independent risk factor for presence of OAB. Additionally, LDL level $\geq 100 \mathrm{~m} / \mathrm{dl}$ was the only other predictive factor for presence of OAB (2.8 times).

Conclusion: The frequency of OAB significantly correlated to severe CAD in postmenopausal women. Moreover, as an additional finding, postmenopausal women with higher LDL levels faced OAB syndrome more frequently.
\end{abstract}

Key Words: Atherosclerosis, Coronary artery disease, Gensini score, Menopause, OAB-V8 form, Overactive bladder.

How to cite this article: Yuksel B, Özgör F, Şahan M, Ozturk S, Can MM, Sarilar O. Correlation between Overactive Bladder Syndrome and Severity of Coronary Artery Disease in Postmenopausal Women. J Coll Physicians Surg Pak 2020; 30(06):622-626 https://doi.org/10.29271/jcpsp.2020.06.622.

\section{INTRODUCTION}

Coronary artery disease (CAD) is the most common cause of death in the developed countries and mortality of CAD patients has been increased by one-third worldwide in the last three decades. ${ }^{1}$ Atherosclerosis (AT) which leads to impaired end othelial function, deteriorated microcirculatory function, increased plaque formation and arterial wall stiffness, is a progressive systemic process of wall of arteries wall and is the main cause of CAD. ${ }^{2}$

Correspondence to: Dr. Faruk Özgör, Haseki Teaching and Research Hospital, Istanbul, Turkey

E-mail:md.farukozgor@gmail.com

Received: April 24, 2020; Revised: June 06, 2020;

Accepted: June 17, 2020

DOI: https://doi.org/10.29271/jcpsp.2020.06.622
It is well known that estrogen has a protective role on development and early progression of AT by improving endothelial functions. Thus, postmenopausal women, who have a lower estrogen level, are more vulnerable to atherosclerotic undesired events. ${ }^{3}$

According to the International Continence Society, overactive bladder syndrome $(\mathrm{OAB})$ is clinical condition that included urgency with or without urgency incontinence, frequency and nocturia in the absence of metabolic and/or pathological disorder that may clarify these symptoms. ${ }^{4}$

Previous studies have found a close correlation between lower urinary symptoms and pelvic ischemia. On the other hand, medium and small sized arteries, such as iliac artery branches, are more sensitive to AT development and ischemic events due to their relatively narrow lumen diameter. Capple et al. empha- 
sised that pelvic ischemia due to AT had a main role in the pathophysiology of lower urinary systems symptoms. ${ }^{5}$ In another study, De et al. found pelvic ischemia by using dynamic contrast-enhanced magnetic resonance imaging in patients with CAD. ${ }^{6}$

Previous studies have demonstrated the association between $C A D$ and pelvic ischemia; however, none of the studies have focused on the relation between the presence of $O A B$ and $C A D$ severity in postmenopausal women, yet. The purpose of the study was to analyse correlation of $O A B$ and severity of $C A D$ with using Gensini score in postmenopausal women.

\section{METHODOLOGY}

After the study was approved by the Local Ethics Committee, female patients with postmenopausal status who had undergone coronary angiography due to CAD in cardiology unit Haseki Training and Research Hospital, Istanbul, Turkey, between January 2016 and January 2018, were enrolled into the study. Informed consent was obtained from all participants and the research was conducted in accordance with Helsinki Declaration. To achieve a more homogenous study population, patients with neurological diseases, pelvic radiation history, urinary system cancers, uncontrolled hypertension and uncontrolled diabetes mellitus were excluded from the study. Also, patients with flow rate $<15 \mathrm{ml} / \mathrm{s}$ and post void residual volume $>$ $100 \mathrm{ml}$ were excluded. Presence of history of pelvic organ surgery, incontinence surgery, history of previous cardiac angiography and cardiac surgery were other exclusion criteria.

Patients who admitted to cardiology outpatient polyclinic with a symptoms or findings (chest pain, stable or unstable angina, coronary artery spasm, repeated acute coronary syndrome and a positive stress test), were examined by a single cardiology specialist. The coronary angiography decision was based on the results of patient history, physical examination findings and imaging modalities outcomes. The Gensini score of each patient was calculated by two different cardiologists separately and the average of two evaluations were taken as the final score. Moreover, all study participants were evaluated about the lower urinary system symptoms and filled the OAB-V8 questionnaire (Validated Turkish form). Age, body mass index, smoking history and serum parameters including fasting glucose, serum creatinine, cholesterol, HDL-cholesterol, LDL-cholesterol, triglyceride levels were recorded. Moreover, urine analysis, urine culture, uroflowmetry and post voiding residual urine volume were performed in each patient. All patients completed the bladderfrequency volume diary.

The participants were separated into two groups according to $\mathrm{OAB}$ symptoms severity. Patients with OAB-V8 score $\geq 8$, were included into group 1 and patients with $O A B-V 8$ score $<8$, were enrolled into group 2, respectively. The groups were compared according to patient's demographic characteristics, Gensini scores, OAB-V8 scores, blood test outcomes, the urinary system ultrasound, and uroflowmetry findings.

The standardised coronary angiography with the Judkin tech- nique was done for all patients and degree of coronary stenosis was assessed by Gensini scoring system. The Gensini score was calculated according to degree of luminal occlusion and its location by two different cardiologists who were blinded to patients' characteristics and laboratory results. The location of stenotic coronary artery were graded as previously described by Gensini which is 5 point for left main system lesions, 2.5 point for proximal left anterior descending artery or proximal circumflex artery lesions, one point for distal left anterior descending artery, mid/distal circumflex artery or right coronary artery lesions, and 0.5 point for lesions in any other artery branches. The score was calculated as sum of the all stenotic coronary artery scores. ${ }^{7}$ The severity of coronary artery stenosis was scored as 1 for $1-25 \%$ narrowing, 2 for $26-50 \%$ narrowing, 4 for $51-75 \%$ narrowing, 8 for $76-90 \%$ narrowing, 16 for $91-99 \%$ narrowing, and 32 for a completely occluded coronary artery.

The OAB-V8 questionnaire is a self-reported form, including 8 questions that assess seriousness of irritative symptoms including frequency, nocturia, urgency and urgency urinary incontinence. The $O A B$ score 8 or more, is meaningfully related with $O A B$, and total $O A B$ score under 8 has shown that diagnosis of $O A B$ is questionable or absent. In OAB-V8 questionnaire, urgency and frequency are described as a sudden sense to voiding and $\geq 8$ voiding per a day, respectively. The nocturia is accepted as waking at night for void $\geq 2$ times and involuntary urine loss with urgency was determined as urgency urinary incontinence. $^{8}$

The Statistical Package of Social Sciences for Windows (SPSS) version 20 was used for statistical analysis. For the analysis of quantitative data, the compliance of data with the normal distribution was checked through the Kolmogorov-Simirnov test. We divided patients into two groups based on OAB-V8 score. Categorical variables were presented as numbers and percentages and compared with Chi-square and Fisher's Exact test. Continuous variables were presented as median (interquartile range) and compared with Mann-Whitney test. Logistic regression (multivariant) analyses were used to examine the possible association between BMI, Gensini score and LDL level. Statistical significance was considered when two-tailed $p$-value $<0.05$.

\section{RESULTS}

During two years of study period, 288 postmenopausal women underwent coronary angiography and 173 patients who accomplished study inclusion criteria, were enrolled into the study. Because of neurological disorders, uncontrolled hypertension, uncontrolled diabetes mellitus, pelvic organ surgery history and usage of medication for OAB; 11, 19, 24, 9 and 38 patients were excluded from the research, respectively. Moreover, 14 patients were not included into the study due to other study exclusion criteria in accordance with the study design.

The 102 (58.96\%) patients with $O A B \geq 8$ score and the 71 (41.04\%) patients with $O A B<8$ score were compared. The age, presence of hypertension and smoking history were similar between two groups $(p=0.141, p=0.742$ and $p=0.692$, respec- 
tively). The median BMI was $28.7(27.3-32.7) \mathrm{Kg} / \mathrm{m}^{2}$ in $\mathrm{OAB}$ group and $27.5(27.0-31.1) \mathrm{Kg} / \mathrm{m}^{2}$ in non-OAB group $(p=0.150)$. Additionally, OAB-V8 score and Gensini score were significantly higher in $\mathrm{OAB}$ group when compared to non-OAB group (25.0 (15.0-28.3) vs. 4.0 (3.0-5.0), $\mathrm{p}<0.001$ and 32.5 (27.8-42.7) vs. 10.0 (10.0-12.0), $p<0.001$, respectively). In blood chemistry, LDL level was the only parameter that was found significantly different between the groups and was significantly higher in patients with OAB (120.0 (90.0-148.5) mg/dl vs. 97.0 (70.0125.0) $\mathrm{mg} / \mathrm{dl}, \mathrm{p}<0.001$ ) (Tablel).

Table I: Comparison of characteristic of patients.

\begin{tabular}{|c|c|c|c|}
\hline & Group & & $n-$ alu \\
\hline & OAB & Non-OAB & p-vatice \\
\hline Number & 102 & 71 & \\
\hline Age (years)* & $58(53-63)$ & $57(53-60)$ & 0.141 \\
\hline BMI $\left(\mathrm{kg} / \mathrm{m}^{2}\right)^{*}$ & $28.7(27.3-32.7)$ & $27.5(27-31.1)$ & 0.150 \\
\hline OAB-V8 score* & $25.0(15-28.3)$ & $4(3-5)$ & $<0.001$ \\
\hline Gensini Score* & $32.5(27.8-42.7)$ & $10(10-12)$ & $<0.001$ \\
\hline Hypertension & $50(49.0 \%)$ & $33(46.5 \%)$ & 0.742 \\
\hline Smoking history & $36(35.3 \%)$ & $23(32.4 \%)$ & 0.692 \\
\hline $\mathrm{LDL}, \mathrm{mg} / \mathrm{ml} *$ & $120(90-148.5)$ & $97(70-125)$ & $<0.001$ \\
\hline $\mathrm{HDL}, \mathrm{mg} / \mathrm{dl} *$ & $46(40.8-54.0)$ & $47(43-51)$ & 0.627 \\
\hline Cholesterol, mg/dl* & $200(166-233.3)$ & $210(170-249)$ & 0.378 \\
\hline Trigliseride, mg/dl* & $146.5(113.8-197.8)$ & $136(107-192)$ & 0.754 \\
\hline Glucose, mg/dl* & $100(92-117)$ & $100(91-119)$ & 0.999 \\
\hline Creatinine, $\mathrm{mg} / \mathrm{dl} *$ & $0.7(0.6-0.7)$ & $0.6(0.6-0.7)$ & 0.530 \\
\hline
\end{tabular}

Table II: Comparison of patients according to OAB symptoms and uroflow parameters between groups.

\begin{tabular}{|l|c|c|c|}
\hline \multirow{2}{*}{} & \multicolumn{2}{|c|}{ Groups } & \\
\cline { 2 - 4 } & OAB & Non-OAB & p-value \\
\hline Number & 102 & 71 & \\
\hline Urgency & $85(83.3 \%)$ & $10(14.1 \%)$ & $<0.001$ \\
\hline Frequency & $71(69.6 \%)$ & $5(7.0 \%)$ & $<0.001$ \\
\hline Uocturia & $92(90.2 \%)$ & $18(25.4 \%)$ & $<0.001$ \\
\hline Postvency uriner incontinance & $59(57.8 \%)$ & $10(14.1 \%)$ & $<0.001$ \\
\hline Max flow rate, $\mathrm{ml} / \mathrm{s}^{*}$ & $30(0-50)$ & $0(0-30)$ & $<0.001$ \\
\hline Average flow rate, $\mathrm{ml} / \mathrm{s}^{*}$ & $26.5(23.5-30)$ & $28(25-31)$ & 0.011 \\
\hline Voided volume, $\left.\mathrm{m}\right|^{*}$ & $9(8-11)$ & $10(9-11)$ & 0.015 \\
\hline Median (interquartile range). & $320(280-380)$ & $350(250-400)$ & 0.522 \\
\hline
\end{tabular}

Table III: Multivariate analysis.

\begin{tabular}{|c|c|c|c|}
\hline & Odds Ratio* & $p * *$ & $p^{* * *}$ \\
\hline Obesity ${ }^{1}$ & $1.8(0.7-4.8)$ & 0.060 & 0.225 \\
\hline $\begin{array}{l}\text { Gensini score } \\
\text { LDL level }^{2}\end{array}$ & $\begin{array}{c}90.8(25.1-329.2) \\
2.8(1.0-7.4)\end{array}$ & $\begin{array}{l}<0.001 \\
0.003\end{array}$ & $\begin{array}{l}<0.001 \\
0.042\end{array}$ \\
\hline \multicolumn{4}{|c|}{$\begin{array}{l}\text { Logistic regression analysis; } 95 \% \text { confidence interval; Univariate analysis; Multivariate analysis; } \\
(1)<30 \mathrm{~kg} / \mathrm{m} 2 \mathrm{vs} \geq 30 \mathrm{~kg} / \mathrm{m}^{2} ;(2)<20 \mathrm{vs} \geq 20 ;(3)<100 \mathrm{mg} / \mathrm{dll} \text { l vs. } \geq 100 \mathrm{~m} / \mathrm{dll} \text {. } \\
\text { CAD: Coronary artery disease; OAB: Overactive bladder syndrome; AT: Atherosclerosis; } \\
\text { BMI: Body mass index. }\end{array}$} \\
\hline
\end{tabular}

In patients with $\mathrm{OAB}$ syndrome, presence of urgency, frequency, and nocturia were determined significantly more common ( $p<0.001, p<0.001$ and $p<0.001$, respectively). Additionally, urgency urinary incontinence rate was higher in patients with $O A B$ syndrome $(57.8 \%$ in patients with $O A B$ and
$14.1 \%$ in patients with non-OAB, $p<0.001$ ). In contrast, patients with non-OAB syndrome had achieved significantly higher the mean maximum flow rate and the mean average flow rate $(28.0$ $(25.0-31.0) \mathrm{ml} / \mathrm{s}$ vs. $26.5(23.5-30.0) \mathrm{ml} / \mathrm{s}, \mathrm{p}=0.011$ and 10.0 $(9.0-11.0) \mathrm{ml} / \mathrm{s}$ vs. $9.0(8.0-11.0) \mathrm{ml} / \mathrm{s}, \mathrm{p}=0.015$, respectively). Group characteristics according to $O A B$ symptoms and uroflowmetry findings were listed in Table II.

Multivariate regression analysis revealed that Gensini score $\geq$ 20 was an independent predictor for presence of $O A B$ syndrome. Additionally, LDL level $\geq 100 \mathrm{~m} / \mathrm{dl}$ was the only other predictive factor for presence of OAB (2.8 times, Table III).

\section{DISCUSSION}

The pathophysiology of OAB has not been fully understood yet. However, a large number of publications suggested that bladder ischemia due to AT is an importantfactor in the development of OAB. Azadzoi et al. demonstrated that bladder ischemia deteriorate mitochondrial functions, decrease protein synthesis/maturation, and stimulate multiple cellular responses including elevated prostaglandin, leukotriene, and transcription factors. ${ }^{9}$ Similarly, Mauri et al. stated that bladder ischemia was associated with inflammation, autophagy, apoptosis, and that process ends with $O A B$ development. ${ }^{10}$

Atherosclerosis is a multifocal and immune inflammatory process of progressive thickening and hardening of arterial wall as a result of fat deposits on their inner lining. Endothelial dysfunction due to AT, results in deteriorated blood flow especially in small and medium-size arteries such as coronary and pelvic arteries. Shaw et al. found that incidence of AT in coronary artery was lower in women but women were faced with a higher incidence of unfavourable outcomes after acute ischemic cardiac events. Moreover, the relatively small size of coronary arteries in women is a factor in decreased success of invasive treatment in women. ${ }^{11}$ Masszi suggested that loss of estrogen protection in postmenopausal period may contribute to the additional risk in development of cardiovascularevents. ${ }^{12}$ Thus, postmenopausal women need special attention in terms of evaluation and treatment of cardiac events.

The diagnosis of CAD has a crucial role to prevent patients' morbidity and mortality; however there is no consensus on appropriate time for evaluation in patients with cardiac risk. Previously, Jackson et al. proved that presence of erectile dysfunction was a sign of CAD. ${ }^{13}$ Additionally, Montorsi et al. suggested that all men with ED should be considered at risk of $\mathrm{CV}$ disease until proven otherwise according to artery size hypothesis. ${ }^{14}$ Due to the systemic effect of AT, CAD and OAB are the two sides of ischemic vascular disorder with the same underlying pathophysiology. In accordance with that hypothesis, we found significant relation between coronary artery stenosis and OAB-V8 score.

Scoring models including coronary anatomy, degree of coronary stenosis, artery morphology have been described for systemic coronary arteries lesions and severity of CAD. Gensini score, which involves coronary artery morphology and degree 
of stenosis, is one of the most frequently used scoring systems for CAD. Chen et al. analysed the Gensini score of 347 patients with $C A D$ and they found significantly higher Gensini score in patients with severe CAD when compared to patients with mild CAD (46.3 vs. 7.1, p<0.001). ${ }^{15}$ In another study by Sinning et al., higher Gensini score was determined as a predictive factor for long term morbidity and mortality in patients with CAD. ${ }^{16}$ Similarly, Gensini score was found significantly higher in patients with severe CAD and OAB symptoms in the present study. Moreover, multivariate regression analysis revealed that Gensini score $\geq 20$ increases the OAB risk.

The LDL is a lipoprotein of blood plasma which composed protein, triglyceride and high cholesterol levels and transports cholesterol from liver to whole body. ${ }^{17}$ The Air Force/Texas Coronary Atherosclerosis Primary Prevention Study (AFCAPS/ TexCAPS) revealed that high LDL levels were precursors for cardiac disorders and $25 \%$ reduction in plasma LDL levels was associated with $37 \%$ reduction of major cardiac events. ${ }^{18}$ In another research, Heart Production Study group followed up 20.536 patients with CAD for mean 5.5 years. Study outcomes demonstrated uneventful cardiac events decreased more than $25 \%$ in patients with baseline LDL-C of less than $100 \mathrm{mg} / \mathrm{dL} .{ }^{19}$ In accordance with high LDL levels-AT-ischemia hypothesis, we found significantly higher LDL levels in patients with $O A B$ and LDL level $\geq 100 \mathrm{mg} / \mathrm{dL}$ may be a predictive factor for OAB.

Previous reports had demonstrated that high intraabdominal and intravesical pressure in obese patients lead to detrusor muscle overactivity and OAB syndrome. In another hypothesis, Richter stated increased abdominal cavity pressure may have a negative effect of pelvic floor innervations. ${ }^{20}$ In another study, Telakova et al. had determined significantly lower ghrelin-A levels, which control the metabolic activity and energy consumption, in obese patients' bladder. ${ }^{21}$ Although, we found higher BMI in patients with $\mathrm{OAB}\left(28.7(27.3-32.7) \mathrm{Kg} / \mathrm{m}^{2}\right.$ vs. 27.5 $\left.(27.0-31.1) \mathrm{Kg} / \mathrm{m}^{2}, \mathrm{p}=0.150\right)$, multivariate regression analysis did not find obesity as predictive factor for OAB $(p=0.225)$. Diverse body types and body fat distribution among humans maylead that outcome in present study.

This study has some limitations. Firstly, present study was a cross-sectional research without long term outcomes. Secondly, study sample included relatively small patient number from single centre. However, to obtain higher internal validity, all participants were examined by same two cardiologists and urology specialist. Additionally, present study did not focus on the interval period between the beginning of OAB symptoms and cardiac symptoms.

\section{CONCLUSION}

The frequency of OAB syndrome was significantly correlated with severe cardiac ischemic disease in postmenopausal women. Moreover, postmenopausal women with higher LDL level more often faced with OAB syndrome. The present study suggested that postmenopausal women with severe coronary artery disease and higher LDL-level should be referred to urology or urogynecology policlinics to heal OAB symptoms and patient's life quality. The present study outcomes must be supported by further prospective studies with a higher patient number.

\section{PATIENTS' CONSENT:}

Informed consents were obtained from all the patients to publish the data concerning this case.

\section{ETHICALAPPROVAL:}

Ethical approval was obtained from Haseki Training and Research Hospital Local Ethical Commitee before the study was conducted in 2016. All procedures performed in studies involving human participants were in accordance with the ethical standards of the institutional and/or national research committee and with the 1964 Helsinki declaration and its later amendments or comparable ethical standards.

\section{CONFLICT OF INTEREST:}

The authors declared no conflict of interest.

\section{AUTHORS' CONTRIBUTION:}

BY: Substantial contributions to the conception or design of the work, agreement to be accountable for all aspects of the work in ensuring that questions related to the accuracy or integrity of any part of the work are appropriately investigated and resolved. Final approval of the version to be published.

FO: Drafting the work or revising it critically for important intellectual content; final approval of the version to be published. SO, MS: The acquisition, analysis.

MMC, OS: Interpretation of data for the work.

\section{REFERENCES}

1. Sanchis Gomar F, Perez Quilis C, Leischik R, Lucia A. Epidemiology of coronary heart disease and acute coronary syndrome. Ann Transl Med 2016; 4(13):256.

2. Herrington $W$, Lacey $B$, Sherliker $P$, Armitage J, Lewington S. Epidemiology of atherosclerosis and the potential to reduce the global burden of atherothrombotic disease. Circ Res 2016; 118(4):535-46.

3. Whayne Jr TF, Mukherjee D. Women, the menopause, hormone replacement therapy and coronary heart disease. Curr Opin Cardiol 2015; 30(4):432-8.

4. Azurı J, Kafri R, Ziv-Baran T, Stav K. Outcomes of different protocols of pelvic floor physical therapy and anticholinergics in women with wet over-active bladder: A 4year follow-up. Neurourol Urodyn 2017; 36(3):755-8.

5. Chapple C. Pathophysiology of neurogenic detrusor overactivity and the symptom complex of "overactive bladder". Neurourol Urodyn 2014; 33(Suppl 3):S6-13.

6. De EJ, Hou P, Estrera AL, Sdringola S, Kramer LA, Graves $\mathrm{DE}$, et al. Pelvic ischemia is measurable and symptomatic in patients with coronary artery disease: a novel application of dynamic contrast-enhanced magnetic resonance imaging. J Sex Med 2008; 5(11):2635-45.

7. Gensini GG. A more meaningful scoring system for determining the severity of coronary heart disease. Am J Cardiol 1983; 51(3):606. 
8. Tarcan T, Mangir N, Ozgur MO, Akbal C. OAB-V8 overactive bladder questionnaire validation study. Üroloji Bülteni 2012; 21:113-6.

9. Azadzoi KM, Shinde VM, Tarcan T, Kozlowski R, Siroky $M B$. Increased leukotriene and prostaglandin release, and overactivity in the chronically ischemic bladder. J Urol 2003; 169(5):1885-91.

10. Maiuri MC, Zalckvar E, Kimchi A, Kroemer G. Self-eating and self-killing: Crosstalk between autophagy and apoptosis. Nat Rev Mol Cell Biol 2007; 8(9):741-52.

11. Shaw LJ, Bugiardini R, Merz CN. Women and ischemic heart disease: evolving knowledge. J Am Coll Cardiol 2009; 54(17):1561-1575.

12. Masszi G. Hypertension and menopause. Orv Hetil 2002; 143:2821-8.

13. Jackson G, Rosen RC, Kloner RA, Kostis JB. The second Princeton consensus on sexual dysfunction and cardiac risk: new guidelines for sexual medicine. J Sex Med 2006; 3(1):28-36.

14. Montorsi P, Ravagnani PM, Galli S, Salonia A, Briganti A, Werba JP, et al. Association between erectile dysfunction and coronary artery disease: Matching the right target with the right test in the right patient. Eur Urol 2006; 50(4): 721-31.

15. Chen ZW, Qian JY, Jian Y, Ge L, Liu XB, Shu XH, et al. Prevalence and severity of coronary artery disease in diabetic patients with aortic valve calcification. Acta Cardiol 2011; 66(1):15-20.
16. Sinning C, Lillpopp L, Appelbaum S, Ojeda F, Zeller T, Schnabel R, et al. Angiographic score assessment improves cardiovascular risk prediction: the clinical value of SYNTAX and Gensini application. Clin Res Cardiol 2013; 102(4): 495-503.

17. Ridker PM. LDL cholesterol: Controversies and future therapeutic directions. Lancet 2014; 384(9943):607-17.

18. Clearfield M, Downs JR, Weis S, Whitney EJ, Kruyer W, Shapiro DR, et al. Air force/texas coronary atherosclerosis prevention study (AFCAPS/TexCAPS): Efficacy and tolerability of long-term treatment with lovastatin in women. J Womens Health Gender Based Med 2001; 10(10):971-81.

19. Baigent C, Landray MJ, Reith C, Emberson J, Wheeler DC, Tomson $C$, et al. The effects of lowering LDL cholesterol with simvastatin plus ezetimibe in patients with chronic kidney disease (Study of Heart and Renal Protection): A randomised placebo-controlled trial. Lancet 2011; 377(9784):2181-92.

20. Richter HE, Creasman JM, Myers DL, Wheeler TL, Burgio KL, Subak LL. Urodynamic characterisation of obese women with urinary incontinence undergoing a weight loss program: The program to reduce incontinence by diet and exercise (PRIDE) trial. Int Urogynecol J 2008; 19(12): 1653-8.

21. Tolekova AN, Hadzhibozheva PV, liev RN, Georgiev CK, Trifonova KY, Sandeva RV, et al. Participation of extracellular $\mathrm{Ca}^{2+}$ or ghrelin in peptide-mediated contraction of strips from rat urinary bladder. Regul Pept 2010; 162(1-3): 79-83. 